\title{
Dualidad director ejecutivo-presidente del directorio, $y$ diferencias en el rol de control del directorio
}

\author{
César E. Freire(1), Tania A. Soto(1), Daniela N. Mendieta(1) y Victor H. Calle(2) \\ (1) Facultad de Ciencias Económicas y Administrativas, Universidad Católica de Santiago de Guayaquil, Guayaquil, \\ Ecuador (correo-e: cesar.freire@cu.ucsg.edu.ec; taniasototobar@gmail.com; danielanmg@hotmail.com) \\ (2) Facultad de Economía Agrícola, Universidad Agraria del Ecuador, Guayaquil, Ecuador. \\ (correo-e: vcalle@uagraria.edu.ec)
}

Recibido Ago. 21, 2019; Aceptado Oct. 16, 2019; Versión final Nov. 7, 2019, Publicado Abr. 2020

\begin{abstract}
Resumen
El presente estudio tuvo por objetivo la determinación de diferencias significativas de la eficacia del control del directorio para empresas que presentan dualidad Director Ejecutivo-Presidente del Directorio y las que no lo presentan. La investigación se desarrolló a través de un enfoque cuantitativo para lo cual se usó un cuestionario para medir la eficacia del control del directorio basado en cuatro dimensiones, esto permitió realizar una prueba de diferencia de medias a través del método del t de student. Como resultado se obtuvo que la Dualidad Director Ejecutivo-Presidente del Directorio incide en los niveles de desempeño de directores independientes y evaluación de desempeño del Director Ejecutivo.
\end{abstract}

Palabras clave: dualidad director-presidente, control de directorio, análisis factorial confirmatorio, eficiencia en el control

\section{Analysis of the duality executive director-chairman of the board, and its relationship with the effectiveness of the control of the board of directors}

\begin{abstract}
The objective of the present study was to determine the differences in the effectiveness of the control of the board of directors for companies that present duality executive director-chairman of the board, and those that do not present this duality. The research is explained through a quantitative approach for which an instrument is used to measure the effectiveness of the control, and is based on four dimensions. The data analysis was done through the test of differences of means using $t$ student. As a result, the duality executive directorchairman of the board influences the performance levels of independent directors and performance evaluation of the executive director.
\end{abstract}




\section{INTRODUCCIÓN}

El objetivo de la presente investigación es determinar diferencias significativas de la eficacia del control del directorio para empresas que presentan dualidad Director Ejecutivo-Presidente del Directorio, siendo la dualidad un fenómeno que se da cuando la misma persona ejerce las dos funciones. Las afectaciones de una organización pueden darse por diversos factores tanto internos como externos. Muchos autores señalan conceptos y teorías muy similares sobre estos temas. Sin embargo, el fin común se basa en la identificación y análisis de dichos factores para que eventualmente las organizaciones puedan aplicar estas teorías y puedan recuperarse de eventualidades fortuitas. El Director Ejecutivo tiene la capacidad suficiente para innovar, reinventar y readecuar su modelo de negocio a tiempo, pero una sola persona no debería hacer el papel de Director Ejecutivo y Presidente del Directorio.

La problemática de la presente investigación radica en que la dualidad Director Ejecutivo - Presidente del directorio es vista como una opción para minimizar costos, sin embargo esto puede afectar al control de la organización. Cuando las empresas optan por esta figura se pone en riesgo la eficacia del control del directorio. En consecuencia es necesario resolver preguntas de investigación dirigidas a esta problemática. La temática resulta relevante dado que las cifras de las empresas que presentan un rol de dualidad del presidente ejecutivo y el presidente del directorio se acercan al $25 \%$ del total de empresas grandes en el Ecuador.

Según el planteamiento de Bass (1985) el liderazgo transformacional aborda que los líderes deben ser guías que orienten a la creatividad y buen manejo no solo de los procesos organizacionales sino también del talento humano. Posteriormente, Nielsen y Munir (2009) señalaron que es imprescindible afrontar un cambio que preserve la sostenibilidad de la organización. Un propósito determina las acciones individuales que deben coordinarse para la obtención de resultados (Pérez, 2017). Silvia et al. (2017) mencionaron la capacidad de los líderes organizacionales refleja el éxito de una compañía. Dicha capacidad se debe potenciar de manera periódica (Martelo et al., 2017). La gestión administrativa del Director Ejecutivo debe ser integral y operativa, lo que transmite claridad en la toma de decisiones y estrategias a considerar. Según Klepper (2010) las personas que pertenecen al directorio o junta de accionistas de la organización tienen bajo su cargo evaluar el desempeño del Director Ejecutivo según sus competencias técnicas y personales.

Hoy en día hablar de gobierno corporativo es un tema en auge, ya que muchas organizaciones empiezan a ponerlo en práctica. Por lo general este tipo de gobierno se ha observado en su mayoría en países de primer mundo. No obstante, en los últimos años países latinoamericanos han optado por adoptar estos modelos por el alto nivel reflejado de desarrollo. Cabe recalcar que las inversiones han aumentado en las empresas del sector privado que ya están manejando este tipo de gobierno evidenciando un crecimiento en aspectos económicos, operacionales, estratégicos, sostenibles y sustentables. Los gobiernos corporativos son un tema relativamente nuevo, a pesar de que sus inicios remontan en la década de 1930, pero el surgimiento de impacto comenzó más tarde en los años 1970 (Subramanian, 2015). El estilo del Director Ejecutivo debe coincidir con el paraje de la empresa, mientras que la junta directiva debe intervenir activamente con él. Mientras que el Presidente del Directorio debe conocer las prácticas de liderazgo de su Director Ejecutivo y las necesidades de la compañía creando el vínculo para que el liderazgo del Director Ejecutivo coincida con las necesidades de esta. Es decir, no se puede contratar el equipo estratégico que trabaje con el Director Ejecutivo si es que no se cumple un proceso eficiente. Este error es el más común y por eso se observa la dualidad entre Director Ejecutivo y el Presidente del Directorio.

Finkelstein y D'aveni (2017) mencionaron que el Director Ejecutivo y el Presidentes del Directorio son diferentes individuos. Cuando existe este tipo de dualidad Director Ejecutivo-Presidente del Directorio se la considera equívoca a pesar de tener un liderazgo fuerte. No obstante, cuando existe dualidad, se genera una especie de seguridad para el Director Ejecutivo de tal forma se puede reguardar de posibles acciones de control de los miembros del directorio. La dualidad Director Ejecutivo-Presidente del Directorio afecta directamente al desempeño de la compañía. Se considera que en empresas grandes las afectaciones provienen en mayor cantidad de los miembros de la junta directiva cuando ésta tiene diversidad de género. De acuerdo con el planteamiento de Ujunwa (2012), en empresas pequeñas se evidenció que la dualidad de la junta y el Director Ejecutivo fue favorable al vincularse positivamente con el origen étnico de los miembros de la junta. Es claro que cuando la junta directiva está activa en el desempeño de sus funciones y cumplimiento de sus deberes, lo esperado es un resultado garantizado de un gobierno corporativo formidable.

Estudios empíricos sobre la dualidad Director Ejecutivo - presidente del directorio revelan resultados conflictivos en la compañía. Una persona que sirve como Director Ejecutivo y Presidente del Directorio puede tener dos únicos resultados: positivo o negativo; ambos causan un alto impacto en el desarrollo organizacional (Peng et al., 2015). Muchas teorías señalan que la dualidad Director Ejecutivo-Presidente del Directorio compromete el monitoreo y control del Director Ejecutivo obteniendo un desempeño totalmente desfavorable. En contraste, las teorías de administración afirman que la dualidad es adecuada si se trata del desempeño 
en la unidad de mando que representa. En países con economías desarrolladas las evidencias palpables son empíricas y en su gran parte son concluyentes y pasan a ser casos de gran interés. En muchos países de América Latina, las principales falencias de los gobiernos corporativos se concentran en los miembros del directorio, estos hacen referencia a la extrema confidencialidad de la información y la falta de cumplimiento en los deberes del Director Ejecutivo y Presidente del Directorio (Penfold et al., 2015).

El tener claro lo que significa éxito es una pauta para la medición de resultados es el principio para definir la eficacia del Director Ejecutivo y Presidente del Directorio. Urriza et al., (2017) añadieron que la importancia de controlar el cumplimiento de los deberes del Director Ejecutivo va de la mano con el análisis de sus objetivos cumplidos y de la evolución, positiva o negativa, que exista. Según Bushman et al. (2004) la eficacia depende de la disponibilidad de información que proporcione el Director Ejecutivo y que transmita al Presidente del Directorio. Puede conceptualizarse que la transparencia corporativa es responsabilidad del Director Ejecutivo, pero al existir dualidad entre los roles se tergiversan los resultados.

Como indica la literatura revisada, en algunos aspectos la dualidad puede ser algo positivo, no obstante, funciona más para compañías pequeñas; este estudio está relacionado directamente con el impacto negativo que obtienen las organizaciones al existir dualidad. Para Samaha et al., (2012) un gobierno corporativo no funciona correctamente cuando existe dualidad Director Ejecutivo-Presidente del Directorio y cuando se contratan directores independientes. La eficacia del directorio se ve comprometida en su totalidad cuando existe dualidad (Briano y Saavedra, 2015). Así mismo Haniffa y Cooke (2002) afirmaron que tanto el Director Ejecutivo como el Presidente del Directorio deben ser personas diferentes, de esta manera mejorará la calidad de la supervisión a las tareas de forma independiente; así mismo se optimizará la divulgación de información corporativa. Cuando existe dualidad los problemas asociados con la falta de intercambio de opiniones son comunes (Haan y Vlahu, 2016).

Por otra parte, Cornett et al. (2010) aseguraron que el Director Ejecutivo tiene menos propiedad y participación que el Presidente Ejecutivo, lo que dificulta la alineación entre los intereses de los gerentes con los accionistas. La junta directiva es el principal instrumento para alinear los intereses dentro de una organización. La orientación empresarial se enfoca a que no exista dualidad del Director Ejecutivo a pesar de que en las economías latinoamericanas se fomenta esta mala práctica debido a instrumentos institucionales, códigos de gobiernos y estándares de gobiernos internacionales (Moser et al., 2019). La evidencia empírica denota que empresas grandes han optado por abandonar la dualidad Director Ejecutivo-Presidente del Directorio a favor de la separación de roles, a fin de mejorar la efectividad de los procesos de la compañía (Linck et al., 2008).

Las empresas cuya estructura de liderazgo es la dualidad de los roles del Director Ejecutivo - Presidente del Directorio, deben optar por cambiar su modelo de liderazgo (Mourthé y Cámara, 2000). Al cambiar a una estructura de roles separados pasan a ser etiquetas como compañías poseedoras de buenas prácticas organizacionales en un gobierno corporativo, lo cual es fundamental para atraer inversionistas (Dey et al., 2011). Así mismo los autores antes mencionados coinciden en que las posiciones de Director Ejecutivo y Presidente del Directorio deben dividirse debido a la presión de los inversionistas, ya que sus contribuciones tienden a ser menores si existe dualidad. Por otra parte, existe la alta sensibilidad al rendimiento de pago en los contratos de compensación del Director Ejecutivo donde tiende a ser significativamente menor al dividir los cargos de Director Ejecutivo y Presidente del Directorio y significativamente mayor cuando existe combinación en estos cargos (Brickley et al., 1997).

La evidencia empírica existente parece apoya los argumentamos de separación de roles sugiere que los costos de separación son menores que los beneficios (Ward et al., 2009). Una característica de las empresas con dualidad Director Ejecutivo-Presidente del Directorio está asociada a un liderazgo de empresas familiares, menos propensas al monitoreo efectivo. Es por eso que se implementan las juntas directivas sólidas e independientes que no han sido contratadas por la administración. De esta manera se asegura un rendimiento mayor y se vela por los intereses de los accionistas (Oh et al., 2018). Arora y Sharma (2016) afirmaron que, el rendimiento sobre el capital y la rentabilidad están relacionados con la dualidad del Director EjecutivoPresidente Ejecutivo, afectando no solo el desempeño sino los índices financieros de las organizaciones. Además, un Director Ejecutivo con una doble función de roles (dualidad) está más inclinado hacia el apalancamiento externo: deuda.

Sobre todo, un Director Ejecutivo con una permanencia más larga tiende a ser oportunista y prioriza su interés personal al tomar decisiones financieras estratégicas, creando así gastos innecesarios (Naseem et al., 2019). Conjuntamente, las características del Director Ejecutivo como edad, género y nivel de estudio (educación) tienen efectos relevantes en las decisiones financieras y el desempeño de la empresa. Finalmente, el indicador de apalancamiento como variable mediadora es significativo para explicar el vínculo que existe entre las características del Director Ejecutivo y el desempeño de la empresa (Haldar et al., 2016). El efecto de la dualidad de los roles del Director Ejecutivo-Presidente del Directorio es negativo cuando el Director Ejecutivo 
tiene un poder dominante en relación a otros ejecutivos y cuando la junta directiva tiene un bloqueo externo, pero no significativo (Tang, 2017). La competitividad de las empresas que presentan una situación de dualidad de roles generan niveles más bajos que aquellas que no comprenden dualidad (Yang y Zhao, 2014). La dualidad del Director Ejecutivo ha sido un detonante en los últimos 20 años, las consecuencias de la dualidad fluctúan dependiendo del giro de negocio de la compañía, pero siempre se resumirán en un mal desempeño organizacional, dejando a la junta directa con poco o ningún control de eficacia e incurriendo en gastos irrelevantes (Krause et al., 2013).

\section{METODOLOGÌA}

El planteamiento de esta investigación se origina de un paradigma conceptual que permite comprender el fenómeno del análisis estudiado, por ende, se aplicó un modelo positivista de lógica deductiva con un enfoque de tipo cuantitativo, de diseño no experimental, debido a que se describe el sujeto de estudio en su contexto natural, sin intervención del investigador. El estudio tiene un alcance correlacional puesto que se analiza la relación entre la dualidad del rol del Director Ejecutivo - Presidente del Directorio y su relación con la eficacia del control del directorio. Una vez realizada la revisión de la literatura, se identificó las dimensiones asociadas con cada una de las variables de estudio. La variable dependiente corresponde a la Eficacia del Control del Directorio la cual se encuentra divida en 4 constructos, para lo cual el instrumento ajustado al estudio contiene 22 preguntas. El cuestionario empleado para la medición de esta variable utiliza una escala de Likert del 1 al 5: (1) Totalmente en desacuerdo, (2) En desacuerdo; (3) Ni de acuerdo, ni en desacuerdo, (4) De acuerdo y (5) Totalmente de acuerdo. Por otra parte, la variable independiente es la Dualidad del rol del Director Ejecutivo - Presidente del Directorio. La encuesta para la cuantificación de la variable mencionada utilizó una pregunta dicotómica de dos alternativas Sí y No.

Tabla 1. Simbología de variables latentes y observadas

\begin{tabular}{|c|c|}
\hline DDI & Desempeño del Directorio Independiente \\
\hline DDI.11 & Habilidades para proveer visiones estratégicas. \\
\hline DDI.12 & Efectividad de representar los intereses de los accionistas. \\
\hline DDI.15 & Compresión sobre la naturaleza del negocio/empresa. \\
\hline DDI.16 & Contribuciones en reuniones con el comité. \\
\hline DDI.17 & $\begin{array}{l}\text { Registro de contribuciones eficientes del directorio y debates constructivos durante las secciones del } \\
\text { directorio. }\end{array}$ \\
\hline DDI.19 & Habilidades al momento de aplicar sus experiencias en la industria. \\
\hline DDI.110 & Comunicarse interactivamente con otros miembros de la junta. \\
\hline $\begin{array}{l}\text { ED-Director } \\
\text { Ejecutivo }\end{array}$ & Evaluación de Desempeño del Director Ejecutivo \\
\hline $\begin{array}{l}\text { ED-Director } \\
\text { Ejecutivo.21 }\end{array}$ & Desempeños obtenidos basados en los resultados de la evaluación. \\
\hline $\begin{array}{l}\text { ED-Director } \\
\text { Ejecutivo.22 }\end{array}$ & Indicadores/medidores de desempeño hacia el Gerente. \\
\hline $\begin{array}{l}\text { ED-Director } \\
\text { Ejecutivo.23 }\end{array}$ & Junta establece un mecanismo de salida vinculado al desempeño del director/Gerente. \\
\hline $\begin{array}{l}\text { ED-Director } \\
\text { Ejecutivo.24 }\end{array}$ & Junta establece un sistema de recompensa (incentivo) basado en el desempeño a largo plazo. \\
\hline $\begin{array}{l}\text { ED-Director } \\
\text { Ejecutivo.25 }\end{array}$ & Junta informa al Gerente sobre las fallas detectadas en función de la evaluación de los resultados. \\
\hline SR-DIR & Supervisión de Riesgo del Directorio \\
\hline SR-DIR.31 & Solicita la junta a la administración general considerar riesgos emergentes que se podrían afrontar. \\
\hline SR-DIR.32 & Directorio recibe actualizaciones por parte de la administración general sobre manejo de riesgo. \\
\hline SR-DIR.33 & Directorio crea conciencia referente al manejo de riesgo. \\
\hline SR-DIR.34 & Junta informa sobre la importancia del buen manejo de riesgos a la administración general. \\
\hline SR-DIR.35 & Directorio asiste a capacitaciones planificadas sobre el manejo de riesgos. \\
\hline SR-DIR.36 & Junta revisa estrategias ante la crisis. \\
\hline AI-DIR & Accesibilidad de información del directorio \\
\hline Al-DIR.41 & Los directivos dialogan a profundidad sobre los asuntos de la empresa. \\
\hline Al-DIR.42 & Los directivos tienen acceso a la información al solicitarla a la gerencia. \\
\hline Al-DIR.43 & $\begin{array}{l}\text { Negativa al acceso de directivos cuando estos necesitan acudir a los registros y libros contables de la } \\
\text { compañía. }\end{array}$ \\
\hline Al-DIR.44 & Requerimiento de asistencia profesional externa de los gastos son solventados por la compañía. \\
\hline
\end{tabular}


Además, se recolectó información dada por las propias empresas para examinar la relación entre las variables que conforman los constructos en análisis (Hernández et al., 2010). Las fuentes de información empleadas fueron primarias debido a que el estudio se recopiló a través del instrumento de recolección de datos; es decir, por medio de cuestionarios debidamente validados por sus respectivos autores. Se empleó un muestreo no probabilístico, estratificado por sectores y dualidad del rol del Director Ejecutivo-Presidente del Directorio con un nivel de muestra de 343 directores independientes con nivel de confianza del $95 \%$ y un margen de error del $5 \%$. Se realizó un análisis de la moda de cada grupo de preguntas con el fin de poder extraer índices que expliquen cada uno de los cuatro constructos analizados sean estos Desempeño del directorio independiente (DDI), Evaluación de desempeño Director Ejecutivo (ED- Director Ejecutivo), Supervisión de riesgo de directorio (SR-DIR), Accesibilidad de información del directorio (AI-DIR).

Los datos recogidos fueron sometidos a diversas pruebas para poder garantizar la robustez de los resultados. Entre las pruebas más representativas están el alfa de Cronbach para explicar su confiablidad, así como supuestos de normalidad univariada como supuesto para aplicar pruebas paramétricas. Posterior a esto se procedió a extraer índices. Los índices extraídos a partir de la moda sirvieron para probar las hipótesis respecto a diferencias de medias. La prueba estadística utilizada fue t para diferencia de medias para muestras independientes, previamente para garantizar robustez se analizó normalidad univariada.

En base al objetivo de investigación se planteó una técnica de tipo correlacional que permitió indicar si existen diferencias significativas entre los niveles de eficacia del control del directorio para empresas que presentan y no presentan un rol de dualidad. La prueba T está diseñada para comparar medias entre dos grupos y es similar al uso de regresión con una variable binaria. En este caso se realiza el contraste de medias para determinar si existe diferencia entre las medias de los cuatro constructos de la eficacia del control del directorio y la dualidad del rol del Director Ejecutivo-Presidente del Directorio. La prueba asume como hipótesis nula que las medias para las dos poblaciones son iguales, para ello se tienen las siguientes hipótesis:

H1a: Si hay diferencia entre el nivel de DDI en empresas con dualidad-sin dualidad Director EjecutivoPresidente del Directorio.

H1b: Si hay diferencia entre el nivel de ED - Director Ejecutivo en empresas con dualidad-sin dualidad Director Ejecutivo-Presidente del Directorio.

H1c: Si hay diferencia entre el nivel de SRDIR en empresas con dualidad-sin dualidad Director EjecutivoPresidente del Directorio.

H1d: Si hay diferencia entre el nivel de AIDIR en empresas con dualidad-sin dualidad Director EjecutivoPresidente del Directorio.

\section{RESULTADOS Y DISCUSIÓN}

Los resultados se basan en la recolección de 343 cuestionarios validados por gerentes o directores de empresas en Ecuador. La presentación de los resultados se estructura de la siguiente manera: (a) exploración de datos, (b) análisis de confiabilidad de las escalas de medición de constructos, y (c) diferencia de medias.

\section{Exploración de Datos}

Para verificar la normalidad univariante, se tomó en cuenta los parámetros de asimetría y curtosis (Hair et al., 2010). Existen diferentes autores que discuten los valores adecuados de estas medidas para determinar la presencia de un problema severo con la distribución. Kline (2011) sostuvo que el valor absoluto en la asimetría mayor que tres y valor de curtosis más de diez pueden indicar un problema, y los valores por encima de 20 pueden indicar un problema más serio. Por lo tanto, el autor sugirió que el valor absoluto en la asimetría y curtosis no debería ser mayor que 3 y 10, respectivamente. Otro criterio es el propuesto por West et al., (2009) que identificaron la no normalidad severa cuando se presentan valores en la asimetría mayores a dos y curtosis mayor a siete. En la Tabla 2 se hizo test de normalidad univariada donde se cuenta que todas las variables del estudio mantuvieron una asimetría y curtosis dentro de los rangos sugeridos (3 y 10 respectivamente) por los autores antes mencionados. Es decir, no existen problemas severos de no normalidad.

\section{Análisis de Confiabilidad}

La confiabilidad puede ser definida como la medida en que una escala obtiene resultados repetibles y que cualquier influencia aleatoria que tienda a hacer mediciones diferentes de una ocasión a otra es una fuente de error de medición, esto indica que el instrumento es consistente. Para evaluar esta condición la herramienta más usada es el Alfa de Cronbach. Este coeficiente provee una medida de la consistencia interna de una escala, se expresa en valores entre cero y uno (Tavakol y Dennick, 2011). Los resultados de esta prueba para cada escala y dimensión se muestran en la Tabla 3. 
Tabla 2. Test de Normalidad Univariada - Asimetría y Curtosis

\begin{tabular}{|c|c|c|}
\hline Variables observadas & Asimetría & Curtosis \\
\hline DDI.11 & $-0,30$ & $-1,12$ \\
\hline DDI.12 & $-1,58$ & 2,08 \\
\hline DDI.15 & $-0,35$ & $-0,71$ \\
\hline DDI.16 & $-2,02$ & 5,45 \\
\hline DDI.17 & $-1,33$ & 4,07 \\
\hline DDI.19 & $-1,70$ & 5,43 \\
\hline DDI.110 & $-1,59$ & 3,67 \\
\hline ED-Director Ejecutivo.21 & $-0,43$ & $-1,12$ \\
\hline ED-Director Ejecutivo.22 & $-0,80$ & $-1,11$ \\
\hline ED-Director Ejecutivo.23 & $-1,07$ & $-0,39$ \\
\hline ED-Director Ejecutivo.24 & $-1,12$ & $-0,39$ \\
\hline ED-Director Ejecutivo.25 & $-1,98$ & 4,47 \\
\hline SR-DIR.31 & $-2,33$ & 5,88 \\
\hline SR-DIR.32 & $-2,66$ & 6,58 \\
\hline SR-DIR.33 & $-2,83$ & 7,11 \\
\hline SR-DIR.34 & $-2,04$ & 3,48 \\
\hline SR-DIR.35 & $-1,61$ & 1,52 \\
\hline SR-DIR.36 & $-2,44$ & 5,19 \\
\hline Al-DIR.41 & $-2,39$ & 7,30 \\
\hline AI-DIR.43 & $-1,74$ & 7,87 \\
\hline Al-DIR.44 & $-0,27$ & $-0,70$ \\
\hline Al-DIR.45 & $-1,96$ & 7,43 \\
\hline
\end{tabular}

Los niveles aceptables para este índice difieren entre varios autores. Valores entre 0,70 a 0,95 son reportados como aceptables por Nunnally y Bernstein (1994); Bland y Altman (1997). Criterios un poco más conservadores sugieren valores entre los rangos 0,80 a 0,90 (Cortina, 1993). De acuerdo con la Tabla 3 se tiene valores en las dimensiones de 0,72 a 0,91 aproximadamente siendo valores aceptables y conservadores para el modelo referente al criterio de los autores. Posterior a la medición de la confiabilidad del cuestionario de investigación, se procedió a realizar la prueba t para muestras independientes, de tal forma se pueda determinar homogeneidad o diferencia de medias. La tabla 4 contiene los valores $\mathrm{p}$ correspondientes a la prueba mencionada. En base a lo indicado, las decisiones de rechazo y no rechazo se realizarán al $5 \%$ y $10 \%$ de significancia

Tabla 3. Coeficiente de Alfa de Cronbach por constructo

\begin{tabular}{|l|l|}
\hline Constructos & Alfa Cronbach \\
\hline DDI & 0,9061 \\
\hline ED-Director Ejecutivo & 0,7235 \\
\hline SR-DIR & 0,7918 \\
\hline Al-DIR & 0,7321 \\
\hline
\end{tabular}

Tabla 4. Prueba T de contraste de media entre los constructos y dualidad del Director Ejecutivo

\begin{tabular}{|l|c|c|c|c|}
\hline \multicolumn{1}{|c|}{ Constructo } & $T$ & $\operatorname{Pr}(T<t)$ & $\operatorname{Pr}(|T|>|t|)$ & $\operatorname{Pr}(T>t)$ \\
\hline DDI & 2,036 & 0,979 & 0,043 & 0,021 \\
\hline ED-Director Ejecutivo & 0,218 & 0,009 & 0,827 & 0,414 \\
\hline SR-DIR & $-2,374$ & 0,586 & 0,012 & 0,991 \\
\hline Al-DIR & $-1,311$ & 0,095 & 0,191 & 0,905 \\
\hline
\end{tabular}


En la Tabla 4 se evidencia que el p-value para los constructos DDI, ED-Director Ejecutivo, SR-DIR y AI-DIR son $0,043,0,827,0,012$ y 0,191 respectivamente y considerando un nivel de significancia de 0,05 se asume que existen diferencias significativas en los constructos DDI y SR-DIR para empresas con y sin Dualidad Director Ejecutivo - Presidente del Directorio. De acuerdo con la Tabla 4, se determinó que los valores $p$ en los constructos SRD y AI-DIR presentan evidencia estadística para no rechazar la hipótesis nula. Por otra parte, en el primer constructo referente a desempeño de directores independientes se tiene un valor $p$ menor al nivel de significancia de 0,05 , lo cual provoca que se rechace la hipótesis nula y no se rechace la hipótesis alternativa que indica diferencia de medias o asociación lineal. Al 0,05 de significancia se rechaza la hipótesis nula y se confirma diferencias de medias para SR-DIR en empresas que presentan y no presentan un rol de dualidad director ejecutivo - presidente del directorio.

De esta manera, se concluye que la variable Dualidad del Director Ejecutivo Presidente del Directorio no se relaciona con la evaluación del desempeño del director ejecutivo y la transparencia de información del directorio, lo cual hace referencia que los propietarios que controlan (sin efecto de la dualidad) a menudo también son los gerentes quienes obtienen el control efectivo de una corporación y tienen el poder de determinar cómo se dirige la compañía y en algunos casos pueden expropiar los derechos de los accionistas minoritarios u trabajadores (Hashim y Devi, 2008). Por otra parte, existe asociación entre la dualidad del rol del Director Ejecutivo-Presidente del Directorio y la supervisión de riesgo y desempeño de directores independientes, de esta forma, Horner (2013) agregó que existe una influencia significativa de la supervisión y accesibilidad de información con la dualidad del rol del Director Ejecutivo-Presidente del Directorio haciendo que se genere un mejor flujo del desarrollo de los procesos internos y comunicaciones en las empresas.

\section{CONCLUSIONES}

Como conclusión principal, la eficacia del control del directorio se diferencia parcialmente en las empresas que presentan y no presentan un rol de dualidad director ejecutivo - presidente del directorio. En base a esto la variable evaluación desempeño de directorio independiente registro diferencias significativas entre empresas que presentan y no presentan un rol de dualidad director ejecutivo - presidente del directorio, además la variable supervisión del riesgo del directorio también incorporo una diferencia aunque con otro significativa. Esta investigación examina la relación de la dualidad del Director Ejecutivo sobre la eficiencia del control del directorio medido por cuatro variables. Las pruebas aplicadas a los modelos indican que no tienen problemas de no normalidad con un ajuste satisfactorio entre variables. Entonces, los resultados indican que la Dualidad del Rol del Director Ejecutivo-Presidente del Directorio íncide en los niveles de desempeño de directores independientes y supervisión de riesgo. Para posteriores estudios se sugiere tener en cuenta los hallazgos dados en la investigación y propagar la importancia en generar este aporte en empresas familiares y pymes mediante preguntas y análisis fundamentales para determinar la eficacia de la junta relacionada a la dualidad Director Ejecutivo Presidente del Directorio.

\section{REFERENCIAS}

Arora, A., y Sharma, C., Corporate governance and firm performance in developing countries: evidence from India. Corporate Governance: The international journal of business in society, 16(2), 420-436 (2016).

Bass, B., Teoría del liderazgo transformacional de Bass. Free Press (1985).

Bland, J., y Altaman, D., Statistics notes: Cronbach's alpha. 572: Bmj (1997).

Briano, G., y Saavedra, M., La composición del consejo de administración y la estructura accionaria como factores explicativos de la transparencia en el gobierno corporativo en Latinoamérica: evidencia en empresas cotizadas de Argentina, Brasil, Chile y México. Estudios Gerenciales, 31(136), 120-130 (2015).

Brickley, J., Coles, J., y Jarrell, G., Leadership structure: Separating the CEO and Chairman of the Board. Journal of Corporate Finance, 3(3), 189-220 (1997).

Bushman, R., Piotroski, J., y Smith, A., What determines corporate transparency? Journal of Accounting Research, 42(2), 207-252 (2004).

Cornett, M., Guo, L., Khaksari, S., y Tehranian, H., Journal of Financial Intermediation, 19(1), 74-94 (2010).

Cortina, J., What is coefficient alpha? An examination of theory and applications. J Appl Psychol, 98-104 (1993).

Dey, A., Engel, E., y Liu, X., CEO and board chair roles: To split or not to split? Journal of Corporate Finance, 17(5), 15951618 (2011).

Finkelstein, S., y D'aveni, R., CEO Duality as a Double-Edge Sword: How Boards of Directors Balance Entrenchment Avoidance and Unity of Command. Academy of Management Journal, 37(5), (2017).

Haan, J., y Vlahu, R., Corporate governance of banks: A survey. Journal of Economic Surveys, 30(2), 228-277 (2016).

Hair, J., Black, W., Babin, B., y Anderson, R., SEM: An introduction. Multivariate data analysis: A global perspective, 629686 (2010). 
Haldar, P. K., Mishra, L., y Dash, G., Corporate Governance Influence on Firm's Financial Performance. Srusti Management Review, 9(2), 33-40 (2016).

Haniffa, R., y Cooke, T., Culture, corporate governance and disclosure in Malaysian corporations. Abacus, 38(3), 317-349 (2002).

Hashim, H., y Devi, S., Board Independence, CEO Duality and Accrual Management. Asian Journal of Business and Accounting, 1(1), 27-46 (2008).

Hernández, R., Fernández, C., y Baptista, P., Metodología de la investigación. México: McGraw Hill (2010).

Horner, S., Board Power, CEO Appointments. Electronic Business Journal, 12(12), 866-878 (2013).

Klepper, W., The CEO's Boss: Tough Love in the Boardroom. Columbia: Columbia University Press (2010).

Kline, R. B., Principles and practice of structural equation modelling (Tercera ed.). New York: Guilford Press (2011).

Krause, R., Semadeni, M., y Cannella, A. Contents Full Article Content List Abstract The CEO Duality-Firm Performance Relationship CEO Duality and Agency Problems The Antecedents of CEO Duality New Directions in CEO Duality Research Conclusion Acknowledgements References Figures y Tables Article Metrics Related Articles Cite Share Request Permissions Explore More Download PDF CEO Duality: A Review and Research Agenda. Journal of Management, 40(1), 256-286 (2013).

Linck, J., Netter, J., y Yang, T., The determinants of board structure. Journal of Financial Economics, 87(2), 308-328 (2008).

Martelo, R., Jiménez, I., y Jaimes, J., Accesibilidad e Integración Digital: Elementos Clave para un Programa de Formación de Empresarios en Empoderamiento Digital. Revista Ciencia y Tecnología, 28(6), 81-94 (2017).

Moser, E., Gama, P., Oliveira, F., y Araki, M., The contextual factors behind CEO Duality: An empirical analysis of Brazil's case. REBRAE: Revista brasilera de estratégia, 12(2), 20-48 (2019).

Mourthé, S., y Câmara, R., Ownership and control structure of brazilian companies. ABANTE, 3(1), 29-56 (2000).

Naseem, M., Lin, J., Rehman, R., Ahmad, M., y Ali, R., Does capital structure mediate the link between CEO characteristics and firm performance? Management Decision (2019).

Nielsen, K., y Munir, F., How do transformational leaders influence followers' affective well-being? Exploring the mediating role of self-efficacy. Work and Stress, 23(4), 313-329 (2009).

Nunnally, J., y Bernstein, I., Psychological theory. New York, NY: MacGraw-Hill (1994).

Oh, W., Chang, Y., y Kim, T., Complementary or Substitutive Effects? Corporate Governance Mechanisms and Corporate Social Responsibility. Journal of Management, 44(7), 2716-2739 (2018).

Penfold, M., Oneto, A., y Guzman, G., Transparency in the Corporate Governance of State-Owned Enterprises in Latin America. Public Policy and Productive Transformation Series, 20, 31-43 (2015).

Peng, M. W., Zhang, S., y Li, X., CEO Duality and Firm Performance during China's Institutional Transitions. Management and Organization Review, 3(2), 205-225 (2015).

Pérez, J., Fundamentos de la dirección de empresas. Colombia: Ediciones RIALP S.A. (2017).

Samaha, K., Dahawy, K., Hussainey, K., y Stapleton, P., The extent of corporate governance disclosure and its determinants in a developing market: The case of Egypt. Advances in Accounting, 28(1), 168-178 (2012).

Silva, O., Toro, L., y Maldonado, F., Resiliencia Organizacional: competencia clave de los CEO. Universidad y Sociedad, 9(5), 165-170 (2017).

Subramanian, G., Corporate Governance 2.0. Harvard Business Review, 97-105 (2015).

Tang, J., CEO duality and firm performance: The moderating roles of other executives and blockholding outside directors. European Management Journal, 35(3), 362-372 (2017).

Tavakol, M., y Dennick, R., Making sense of Cronbach's alpha. International journal of medical education, 2-53 (2011).

Ujunwa, A., Board characteristics and the financial performance of Nigerian quoted firms. Corporate Governance: The international journal of business in society, 12(5), 656-674 (2012).

Urriza, M., Manzanal, M., Acuña, A., y Subota, L., Gobernabilidad y Gobierno Corporativo: Análisis y medición de su eficacia en organizaciones. Revista de ADENAG (2017).

Ward, A. J., Brown, J. A., y Rodriguez, D., Governance Bundles, Firm Performance, and the Substitutability and Complementarity of Governance Mechanisms. Corporate Governance: An International Review, 17(5), $646-660$ (2009).

West, F., y Chiou, H., Multilevel moderated mediation of organizational study: An empirical analysis of organizational innovation climate, organizational commitment, and job satisfaction. Journal of Management, 26(2), 189-211 (2009).

Yang, T., y Zhao, S., CEO duality and firm performance: Evidence from an exogenous shock to the competitive environment. Journal of Banking y Finance, 49, 534-552 (2014). 